OPEN ACCESS

Edited by:

Ivan Mijakovic,

Chalmers University of Technology

Sweden

Reviewed by:

Antoine Danchin,

Institut de Cardiométabolisme et

Nutrition (ICAN), France

Natalia Tschowri,

Humboldt University of Berlin,

Germany

*Correspondence:

Jörg Stülke

jstuelk@gwdg.de

tThese authors have contributed equally to this work.

Specialty section:

This article was submitted to Microbial Physiology and Metabolism,

a section of the journal

Frontiers in Microbiology

Received: 13 April 2017 Accepted: 30 June 2017

Published: 13 July 2017

Citation:

Blötz C, Treffon K, Kaever V, Schwede F, Hammer E and Stülke J

(2017) Identification of the

Components Involved in Cyclic Di-AMP Signaling in Mycoplasma pneumoniae.

Front. Microbiol. 8:1328. doi: 10.3389/fmicb.2017.01328

\section{Identification of the Components Involved in Cyclic Di-AMP Signaling in Mycoplasma pneumoniae}

\author{
Cedric Blötz ${ }^{1 \dagger}$, Katrin Treffon ${ }^{1 \dagger}$, Volkhard Kaever ${ }^{2}$, Frank Schwede ${ }^{3}$, Elke Hammer ${ }^{4}$ and \\ Jörg Stülke ${ }^{\text {* }}$
}

1 Department of General Microbiology, Institute of Microbiology and Genetics, Georg-August University Göttingen, Göttingen, Germany, ${ }^{2}$ Research Core Unit Metabolomics, Hannover Medical School, Hannover, Germany, ${ }^{3}$ Biolog Life Science Institute, Bremen, Germany, ${ }^{4}$ Department of Functional Genomics, Interfaculty Institute for Genetics and Functional Genomics, University Medicine Greifswald, Greifswald, Germany

Bacteria often use cyclic dinucleotides as second messengers for signal transduction. While the classical molecule c-di-GMP is involved in lifestyle selection, the functions of the more recently discovered signaling nucleotide cyclic di-AMP are less defined. For many Gram-positive bacteria, c-di-AMP is essential for growth suggesting its involvement in a key cellular function. We have analyzed c-di-AMP signaling in the genome-reduced pathogenic bacterium Mycoplasma pneumoniae. Our results demonstrate that these bacteria produce c-di-AMP, and we could identify the diadenylate cyclase CdaM (MPN244). This enzyme is the founding member of a novel family of diadenylate cyclases. Of two potential c-di-AMP degrading phosphodiesterases, only PdeM (MPN549) is active in c-di-AMP degradation, whereas NrnA (MPN140) was reported to degrade short oligoribonucleotides. As observed in other bacteria, both the c-di-AMP synthesizing and the degrading enzymes are essential for $M$. pneumoniae suggesting control of a major homeostatic process. To obtain more insights into the nature of this process, we have identified a c-di-AMP-binding protein from M. pneumoniae, $\mathrm{KtrC}$. KtrC is the cytoplasmic regulatory subunit of the low affinity potassium transporter KtrCD. It is established that binding of c-di-AMP inhibits the KtrCD activity resulting in a limitation of potassium uptake. Our results suggest that the control of potassium homeostasis is the essential function of c-di-AMP in M. pneumoniae.

Keywords: second messenger, diadenylate cyclase, phosphodiesterase, c-di-AMP, potassium uptake, Mollicutes

\section{INTRODUCTION}

To respond to changes in their environment, bacteria have evolved a large set of signal detection and transduction systems. Such signals can be directly sensed by proteins, they may feed into complex networks that control gene expression via transcription factors, or they are first converted to so-called second messengers which in turn provoke a response. Many bacteria use dedicated nucleotides as second messengers, among them cyclic AMP, (p)ppGpp and the cyclic dinucleotides c-di-AMP and c-di-GMP (Gomelsky, 2011). 
cAMP is the paradigmatic second messenger, and this nucleotide is involved in coordinating carbon and nitrogen metabolism and in carbon catabolite repression in Escherichia coli and related bacteria (Görke and Stülke, 2008; You et al., 2013). (p)ppGpp is formed upon starvation and triggers a reduction of cellular house-keeping activities (Steinchen and Bange, 2016). Cyclic di-GMP is in many bacteria involved in the choice between sessile and motile lifestyles (Hengge, 2009). While these second messengers have been intensively studied in a large number of different bacteria, cyclic di-AMP has been discovered only less than a decade ago (Witte et al., 2008). This nucleotide was found in the crystal structure of the so-called DNA integrity scanning protein DisA, which exhibits diadenylate cyclase activity (Witte et al., 2008). C-di-AMP is formed in a variety of both Gram-positive and Gram-negative bacteria with the notable exception of the gamma-proteobacteria including the enterobacteria. Several studies have revealed that this second messenger is essential for many Gram-positive bacteria with a low genomic GC content (the Firmicutes). This has been shown for Bacillus subtilis, Staphylococcus aureus, Listeria monocytogenes, and many other species (Luo and Helmann, 2012; Corrigan and Gründling, 2013; Commichau et al., 2015). Moreover, the accumulation of c-di-AMP has also been shown to cause severe problems for the cells (Mehne et al., 2013; Gundlach et al., 2015b). To control the intracellular levels of c-di-AMP, the bacteria that produce cyclic di-AMP do also possess phosphodiesterases to degrade this molecule (Rao et al., 2010; Corrigan et al., 2011; Commichau et al., 2015; Huynh and Woodward, 2016).

All known diadenylate cyclases share a conserved domain, the so-called DAC domain (Römling, 2008; Commichau et al., 2015; Rosenberg et al., 2015). However, the DAC domain can be found in different arrangements with other domains. Based on the domain organization, three classes of diadenylate cyclases have been studied so far (see Figure 1). The proteins of the $\mathrm{CdaA}$ class are membrane proteins with three transmembrane domains at the N-terminus (Gundlach et al., 2015b). This class of diadenylate cyclases is the most widespread, and it is ubiquitous in most Firmicutes. CdaS is a cyclase that is only found in B. subtilis and closely related spore formers. This cyclase consists of an N-terminal autoinhibitory domain and the DAC domain (Mehne et al., 2014). Finally, the enzymes of the DisA class are found in spore-forming Firmicutes (Bacillus spp., Clostridium spp.) and in the Actinobacteria. These octameric enzymes have their DAC domain at the N-terminus, and bind DNA via a C-terminal helix-hairpin-helix domain (Witte et al., 2008; Commichau et al., 2015). While most bacterial species contain one diadenylate cyclase, $B$. subtilis encodes three enzymes, one of each class (Mehne et al., 2013). Two principal classes of c-diAMP-degrading phosphodiesterases have been described: The proteins of the first class possess a domain called DHH-DHHA1 with a catalytic Asp-His-His motif. The DHH-DHHA1 domain can either be part of a larger protein as in B. subtilis GdpP or exert the enzymatic activity without any additional domains as described for Streptococcus pneumoniae Pde2 (see Figure 1, Rao et al., 2010; Bai et al., 2013). The enzymes of the second class possess a so-called $\mathrm{HD}$ domain with a His-Asp catalytic motif (Huynh et al., 2015).
While the functions of cAMP, (p)ppGpp, and c-di-GMP are well understood, this is not the case for c-di-AMP. Earlier studies have implicated c-di-AMP in the control of cell wall homeostasis (Corrigan et al., 2011; Luo and Helmann, 2012; Mehne et al., 2013). Moreover, c-di-AMP formation by DisA was suggested to be important for integrity control, repair and recombination of DNA (Oppenheimer-Shaanan et al., 2011; Campos et al., 2014). The isolation of c-di-AMP binding proteins has identified the small signal transduction protein DarA, the pyruvate carboxylase, subunits of potassium transporters, and ATP-binding subunits of osmoprotectant ABC transporters (Corrigan et al., 2013; Sureka et al., 2014; Gundlach et al., 2015a; Huynh et al., 2016; Schuster et al., 2016). Moreover, c-di-AMP binds and inhibits the KdpD sensor kinase that controls the expression of a highaffinity potassium transporter in S. aureus (Moscoso et al., 2016). Finally, c-di-AMP binds an RNA molecule, i.e., the riboswitch that controls the expression of the potassium transporters KimA and KtrAB in B. subtilis (Nelson et al., 2013; Gundlach et al., 2017). Binding to both KtrA and the riboswitch controlling its expression makes c-di-AMP the only second messenger that controls a biological process (potassium uptake) by binding both to a protein and to the corresponding mRNA molecule (Commichau et al., 2015). Among all identified targets of c-diAMP, not a single one is essential. Only recently, c-di-AMP essentiality could be traced back to the control of potassium homeostasis in B. subtilis (Gundlach et al., 2017).

We are interested in signal transduction in the strongly genome-reduced pathogenic bacterium Mycoplasma pneumoniae. This bacterium encodes only 694 proteins (Lluch-Senar et al., 2015), reflecting its adaptation to rather constant environmental conditions in the natural habitat, human lung epithelia. Accordingly, M. pneumoniae possesses only three putative transcription factors to control gene expression. However, the mechanisms of transcriptional regulation in M. pneumoniae are still poorly understood (Güell et al., 2009). In addition, the second messenger ppGpp is likely formed by $M$. pneumoniae as deduced from the presence of a ppGpp synthetase-encoding gene (Eilers, 2010).

In this study, we have analyzed the presence of components involved in c-di-AMP signaling in $M$. pneumoniae. We demonstrate that this second messenger is formed by this bacterium, and have identified the enzymes responsible for its synthesis and degradation. Moreover, we have discovered that c-di-AMP binds to the M. pneumoniae KtrC protein, indicating a function in the control of potassium uptake.

\section{MATERIALS AND METHODS}

\section{Bacterial Strains and Growth Conditions}

Mycoplasma pneumoniae was handled under L2 laboratory safety conditions. The M. pneumoniae strain used in this study was M. pneumoniae M129 (ATCC 29342). M. pneumoniae was grown at $37^{\circ} \mathrm{C}$ in $175 \mathrm{~cm}^{2}$ tissue culture flasks containing $100 \mathrm{ml}$ of modified Hayflick medium as described previously (Halbedel et al., 2004). Carbon sources were added to a final concentration of $1 \%(\mathrm{w} / \mathrm{v})$. Escherichia coli XL1blue and BL21(DE3)/pLysS 
A

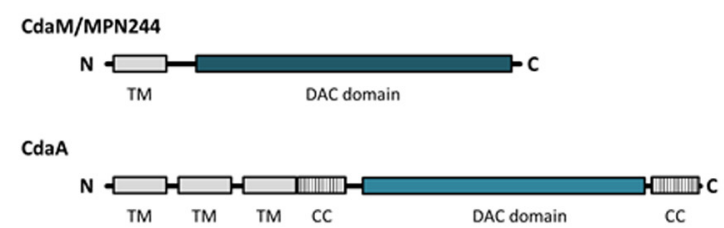

Cdas

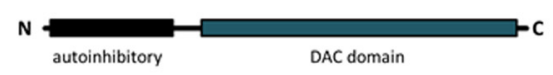

DisA

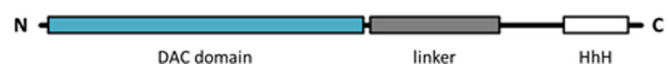

B
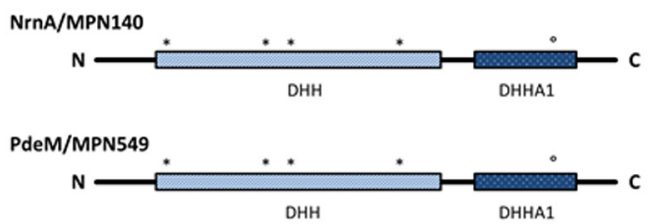

GdpP

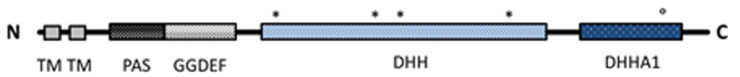

Pde2

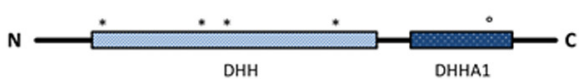

FIGURE 1 | Domain architecture of c-di-AMP related enzymes. Black lines indicate protein sequences and the boxes represent domains. (A) The four classes of C-di-AMP producing DACs. Intensity of the blue color indicates differences in the DAC domain. (B) Phosphodiesterases specific for C-di-AMP degradation. Conserved residues in the $\mathrm{DHH}$ and DHHA1 motifs are indicated with asterisks and circle, respectively. DAC, di-adenylate cyclase; TM, transmembrane domain; CC, coiled-coil domain; HhH, Helix-hairpin-Helix; PAS, Per-Arnt-Sim sensor domain; DHH/DHHA/GGDEF, Asp-His-His/ Asp-His-His-Ala/ Gly-Gly-Asp-Glu-Phe.

(Sambrook et al., 1989) were used as host for cloning and recombinant protein expression, respectively. E. coli strains were cultivated in Luria Bertani broth (Sambrook et al., 1989) containing ampicillin $100 \mu \mathrm{g} \mathrm{ml}^{-1}$. Bacillus subtilis 168 was used to extract chromosomal DNA.

\section{DNA Manipulation and Plasmid Construction}

Chromosomal DNA from B. subtilis and $M$. pneumoniae was isolated using the Blood and Tissue Kit according to the manufacturer's instructions (Qiagen, Hilden, Germany). Transformation of E. coli and plasmid DNA extraction was performed using standard procedures (Sambrook et al., 1989).

Plasmids for the overexpression and purification of the enzymes potentially involved in c-di-AMP metabolism from M. pneumoniae were constructed as follows. The coding sequence of each gene was amplified by PCR with gene specific primers (listed in Supplementary Table S1) using chromosomal DNA of M. pneumoniae M129 as the template. As the M. pneumoniae genes contain TGA codons that code for tryptophan in M. pneumoniae but for an opal stop codon in E. coli, the PCR fragments were used as templates for mutagenesis by the multiple mutation reaction (Hames et al., 2005) using the amplification primers and $5^{\prime}$-phosphorylated mutagenic primers (listed in Supplementary Table S1) to introduce TGA to TGG transitions. The cytoplasmic portion of the B. subtilis $g d p P$ gene $\left(\mathrm{GdpP}_{84-659}\right)$ was amplified from $B$. subtilis chromosomal DNA. The PCR products of genes potentially encoding phosphodiesterases were digested with BamHI and NdeI and cloned into the expression vector pGP574 (Schilling et al., 2006). This plasmid allows the expression of enzymes carrying a C-terminal Strep-tag. The resulting plasmids are pGP2717 (mpn140), pGP2718 (mpn549), and pGP2720 ( $g d p P$ ). The PCR product for the potential diadenylate cyclase-encoding gene mpn244 was cleaved with NdeI and BamHI and cloned into the vector pET3c (Novagene, Darmstadt, Germany). The resulting plasmid was pGP2036. All plasmid inserts were verified by DNA sequencing.

\section{Protein Overexpression and Purification}

The Strep-tagged proteins were overexpressed in E. coli BL21(DE3). Expression ( 11 culture, $37^{\circ} \mathrm{C}, 200 \mathrm{rpm}$, baffled flasks) was induced by the addition of $1 \mathrm{mM}$ IPTG to exponentially growing cultures $\left(\mathrm{OD}_{600}\right.$ of 0.6 to 0.8$)$. After expression for 3 hours, the cells were pelleted at $4^{\circ} \mathrm{C}$ for $20 \mathrm{~min}$ at $4,000 \mathrm{rpm}$ and washed once with $20 \mathrm{ml}$ PDE buffer $(100 \mathrm{mM}$ Tris- $\mathrm{HCl}$ pH8.3, $150 \mathrm{mM} \mathrm{NaCl}, 1 \mathrm{mM}$ EDTA, $5 \%$ glycerol). Cells were lysed using a French press (18,000 p.s.i., 138,000 kPa, three passes, SLM Aminco, United States). After lysis, the crude extracts were centrifuged at 35000 r.p.m for $30 \mathrm{~min}$. The crude extract was then passed over a StrepTactin column (IBA, Göttingen, Germany). The recombinant proteins were eluted with desthiobiotin (IBA, final concentration $2.5 \mathrm{mM}$ ). After elution, the fractions were tested for the desired protein using 12\% SDS-PAGE. Only fractions which contained the desired protein in apparent homogeneity (content of the specific protein $>95 \%$ ) were used for further purification by running the proteins over a $10 \mathrm{ml}$ HiTrap Heparin HP column (GE Healthcare) using the ÄKTA prime plus system (GE Healthcare, flow rate $2 \mathrm{ml} / \mathrm{min}$ ). With $1 \mathrm{M} \mathrm{NaCl}$ in the PDE buffer, proteins were eluted from the column. Fractions containing pure protein were concentrated and dialyzed to $1.5 \mathrm{ml}$ without $\mathrm{NaCl}$ in $\mathrm{PDE}$ buffer using Vivaspin ${ }^{\circledR}$ Turbo15 ultrafiltration spin columns (MW 5,000 Da; Sartorius, Göttingen, Germany). Protein concentrations were determined using the Bio-Rad dye-binding assay where bovine serum albumin served as the standard. Aliquots of the proteins were frozen in liquid nitrogen and stored at $-80^{\circ} \mathrm{C}$ prior to further experiments.

\section{Enzyme Assays}

The assays for phosphatase and phosphodiesterase activities of Strep-tagged proteins were performed as described previously 
(Diethmaier et al., 2014). Phosphatase activity against paranitrophenol phosphate ( $p$ NPP) was assayed in a buffer containing $100 \mathrm{mM}$ Tris- $\mathrm{HCl}$ pH8.3, $10 \mathrm{mM} \mathrm{NaCl}, 0.1 \mathrm{mM} \mathrm{MnCl}_{2}, 25 \mathrm{mM}$ $p \mathrm{NPP}$ and different amounts of purified protein in a total volume of $100 \mu \mathrm{l}$. Phosphodiesterase activity against bis- $p$ NPP was assayed in a buffer containing $100 \mathrm{mM}$ Tris- $\mathrm{HCl} \mathrm{pH} 8.3$, $10 \mathrm{mM} \mathrm{NaCl}, 0.1 \mathrm{mM} \mathrm{MnCl}_{2}$, or $\mathrm{MgCl}_{2}, 1-7.5 \mathrm{mM}$ bis- $p \mathrm{NPP}$ and purified protein in a total volume of $100 \mu \mathrm{l}$. The reactions were initiated by the addition of the protein, and the reaction mixture was incubated at $37^{\circ} \mathrm{C}$ for $4 \mathrm{~h}$. Relative substrate cleavage was quantified by measuring the $\mathrm{OD}_{410}$ using a microplate reader (EPOCH| 2, BioTek, Winooski, United States).

Phosphodiesterase activity with cyclic dinucleotides was measured using a quantitative assay based on the interaction of c-di-AMP with the fluorescent dye coralyne (Zhou et al., 2014). Briefly, reaction mixtures $(150 \mu \mathrm{l})$ consisted of $100 \mathrm{mM}$ Tris- $\mathrm{HCl}$ pH8.3, $10 \mathrm{mM} \mathrm{NaCl}, 0.1 \mathrm{mM} \mathrm{MnCl}_{2}$, and $100 \mu \mathrm{M}$ c-di-AMP. The reaction was initiated by addition $100 \mathrm{nM}$ enzyme (for MPN549 additionally 50 and $10 \mathrm{nM}$ ), and the reaction mixture was incubated at $37^{\circ} \mathrm{C}$ for $4 \mathrm{~h}$. At different time points the reactions were stopped by addition of $1 \mu$ EDTA $(0.5 \mathrm{M})$, shockfreezing and subsequent boiling for $10 \mathrm{~min}$. To quantify the remaining c-di-AMP, the samples were spun to remove protein precipitate. $\mathrm{KBr}$ and coralyne were added to the sample to final concentrations of $250 \mathrm{mM}$ and $10 \mu \mathrm{M}$, respectively. After $20 \mathrm{~min}$ of incubation at $37^{\circ} \mathrm{C}$ in the dark, relative c-di-AMP cleavage was determined as described (Zhou et al., 2014) by measuring the fluorescence emission at $475 \mathrm{~nm}$ using a microplate reader (SYNERGY Mx, BioTek, Winooski, United States).

In all kinetic experiments, $K_{\mathrm{m}}$ and $V_{\max }$ were determined by nonlinear curve fitting from Lineweaver-Burk plots.

\section{c-di-AMP Extraction}

The concentrations of c-di-AMP in E. coli and in M. pneumoniae cells were determined by a liquid chromatography tandem MS (LC-MS/MS) method, essentially as described previously (Mehne et al., 2013; Gundlach et al., 2015b). Briefly, E. coli or M. pneumoniae cells were grown in LB $(20 \mathrm{ml})$ or MP $(100 \mathrm{ml})$ medium, respectively. For E. coli two additional aliquots $(1 \mathrm{ml}$ each) of each sample were harvested for total protein determination. For M. pneumoniae, the wet weight of each sample was determined. The pellets were resuspended in $300 \mu \mathrm{l}$ extraction mixture (acetonitrile/methanol/water 40/40/20 $\mathrm{v} / \mathrm{v} / \mathrm{v}$ ) and shock-frozen in liquid nitrogen, followed by boiling for $10 \mathrm{~min}$. The boiled samples were centrifuged for $10 \mathrm{~min}$ at $4^{\circ} \mathrm{C}$ and $20,800 \mathrm{~g}$. The supernatants were stored on ice, and the remaining pellets were used for two more extraction steps with $200 \mu \mathrm{l}$ extraction mixture. For this purpose, the samples were mixed, incubated on ice for $15 \mathrm{~min}$, and centrifuged again. The obtained supernatants were pooled. The samples were incubated at $-20^{\circ} \mathrm{C}$ over night, and centrifuged again $\left(20 \mathrm{~min}, 4^{\circ} \mathrm{C}, 20,800 \mathrm{~g}\right)$. Then, the supernatant was transferred to a fresh reaction tube, and dried in a speed vac at $50^{\circ} \mathrm{C}$ for $2 \mathrm{~h}$ and resuspended in $200 \mu \mathrm{l}$ of water. After repeated centrifugation and addition of the internal standard $\left(\left[{ }^{13} \mathrm{C},{ }^{15} \mathrm{~N}\right] \mathrm{c}\right.$-di-AMP), part of the extract was analyzed by LC-MS/MS.

\section{Quantification of c-di-AMP by MS/MS}

Chromatographic separation was performed on a series 200 high-pressure liquid chromatography (HPLC) system (PerkinElmer Life Sciences) as described previously (Mehne et al., 2013). The analyte detection was performed on an PI4000 triple-quadrupole mass spectrometer equipped with an electrospray ionization source (AB Sciex), using selected reaction monitoring (SRM) analysis in positive ionization mode. The SRM transitions labeled "quantifier" were used to quantify the compound of interest, whereas "identifier" SRM transitions were monitored as confirmatory signals. The quantifier SRM transitions were most intense and were therefore used for quantification.

\section{Pull Down of c-di-AMP Binding Proteins Via c-di-AMP Coupled Agarose}

To isolate c-di-AMP-binding proteins from $M$. pneumoniae, we made use of immobilized c-di-AMP (2'-O-(6aminohexylcarbamoyl)-cyclic diadenosine monophosphate on agarose (2'-AHC-c-di-AMP-agarose; BIOLOG, Bremen, Germany). We prepared $M$. pneumoniae crude cell extracts from $150 \mathrm{mg}$ cells per assay. The cells were lysed in pulldown buffer $(100 \mathrm{mM}$ Tris- $\mathrm{HCl} \mathrm{pH} 7.5,100 \mathrm{mM} \mathrm{KCl}, 150 \mathrm{mM}$ $\mathrm{NaCl}, 5 \mathrm{mM} \mathrm{MgCl}$, $0.5 \mathrm{mM} \mathrm{DTT}, 0.1 \%$ (v/v) Tween-20, and $1 \mathrm{x}$ cOmplete ${ }^{\mathrm{TM}}$ protease inhibitor (Roche Diagnostics, Mannheim, Germany)). Cells were lysed in a tissue lyser with $0.1 \mathrm{~mm}$ glass beads $(2 \times 2.5 \mathrm{~min}, 30 \mathrm{~Hz}$, cooled block $)$ followed by ultracentrifugation for $30 \mathrm{~min}$ at $35,000 \mathrm{rpm}$ and $4^{\circ} \mathrm{C}$. To prepare the matrix, $500 \mu \mathrm{l} 2^{\prime}$-AHC-c-di-AMP agarose or ethanolamine-coupled agarose (negative control) were equilibrated three times with $1 \mathrm{ml}$ of pulldown buffer. After equilibration, the cell lysate and the agarose matrix were incubated rotating over night at $4^{\circ} \mathrm{C}$. The supernatant was discarded and the matrix was washed three times $(1 \mathrm{ml}$ 1x PBS; $137 \mathrm{mM} \mathrm{NaCl}, 2.7 \mathrm{mM} \mathrm{KCl}, 10 \mathrm{mM} \mathrm{Na} \mathrm{HPO}_{4}$, $1.76 \mathrm{mM} \mathrm{KH} \mathrm{PO}_{4}, \mathrm{pH} 7.4$ ). Elution of binding proteins was performed with $200 \mu \mathrm{l}$ of $1 \mathrm{x}$ PBS, pH7.4 containing $1 \mathrm{mM}$ c-di-AMP and $15 \mathrm{~min}$ incubation at RT and 1,400 rpm. The eluted proteins were concentrated using acetone precipitation (1:1 sample:acetone) over night at $-20^{\circ} \mathrm{C}$. The precipitate was centrifuged $1 \mathrm{~h}$ at $14,800 \mathrm{rpm}$ and $4^{\circ} \mathrm{C}$. The remaining supernatant was discarded and the sample dried for $10 \mathrm{~min}$ at RT. The remaining pellet was solved in $40 \mu \mathrm{l} 1 \mathrm{x}$ PBS and analyzed by SDS-PAGE. Proteins of interest were identified by mass spectrometry as described previously (Meyer et al., 2011). Briefly, after destaining of gel slices, proteins underwent in-gel digestion with trypsin (10 $\mathrm{ng} / \mu \mathrm{l}$ trypsin in $20 \mathrm{mM}$ ammonium bicarbonate). Peptides were separated by $\mathrm{C} 18$ reverse phase liquid chromatography (nanoAcquity UPLC system, $10 \mathrm{~cm}$, Waters, Manchester, United Kingdom) in a linear gradient of $0.1 \%$ acetic acid in acetonitrile from $5 \%$ up to $25 \%$ within $65 \mathrm{~min}$ (flow rate: $400 \mathrm{nl} / \mathrm{min}$ ). MS analysis was performed on a LTQ-Orbitrap Velos hybrid mass spectrometer (Thermo Electron, Bremen, Germany) operated in data-dependent MS/MS mode. Proteins were identified by searching all MS/MS spectra against a M. pneumoniae M129 protein database (687 
entries, extracted from Uniprot rel. 05-2014) using Sequest algorithm on a SorcererTM software platform. Initial mass tolerance for peptide identification on MS and MS/MS peaks were $10 \mathrm{ppm}$ and $1 \mathrm{Da}$, respectively. Up to two missed tryptic cleavages were allowed. Methionine oxidation $(+15.99492 \mathrm{Da})$ and propionamide modification on cysteine (+71.037109 Da) were set as variable modifications. Protein identification results were evaluated by determination of probability for peptide and protein assignments provided by PeptideProphet and ProteinProphet (ISI Seattle, WA, United States) incorporated in the Scaffold software package rel. 4.3.2 (Proteome Software, Portland, OR, United States). Proteins were identified by at least two peptides with peptide probability $>90 \%$ reflecting protein probability of $>99 \%$.

\section{Assay for M. pneumoniae Mutants}

For the isolation of $M$. pneumoniae mutants, we used an ordered collection of strains carrying insertions of transposon Tn4001 (Halbedel et al., 2006). The presence of the desired mutant was assayed by a PCR screen using one primer that hybridizes to the transposon (directed outward), and a second primer specific for the gene of interest (see Supplementary Table S1).

TABLE 1 | Kinetic parameters for phosphodiesterase activity on bis-pNPP.

\begin{tabular}{|c|c|c|c|c|}
\hline \multirow[t]{2}{*}{ Protein } & \multicolumn{2}{|c|}{$K_{\mathrm{m}}[\mathrm{mM}]$} & \multicolumn{2}{|c|}{$V_{\max }\left[\mu \mathrm{mol} \mathrm{min}{ }^{-1} \mathrm{mmol}^{-1}\right]$} \\
\hline & $+\mathrm{MnCl}_{2}$ & $+\mathrm{MgCl}_{2}$ & $+\mathrm{MnCl}_{2}$ & $+\mathrm{MgCl}_{2}$ \\
\hline MPN140 & 1.60 & 2.90 & 0.22 & 0.19 \\
\hline MPN549 & 0.60 & 0.55 & 0.04 & 0.04 \\
\hline GdpP & 1.45 & 1.64 & 0.02 & 0.02 \\
\hline
\end{tabular}

\section{RESULTS}

\section{M. pneumoniae Proteins Potentially Involved in c-di-AMP Metabolism}

All Firmicutes studied so far produce c-di-AMP, and in most of them, this second messenger is essential for the growth of these bacteria (Commichau et al., 2015). However, the presence of this signaling nucleotide has never been analyzed in representatives of the Mollicutes. To address this question for $M$. pneumoniae, we cultivated cells, extracted nucleotides, and analyzed these extracts for the presence of c-di-AMP. The total amount of c-di-AMP (nM) extracted from the pellet was divided by the total cell volume $(\mathrm{mg})$ to yield the c-di-AMP concentration per mg cells. In three independent biological experiments (with three technical replicates each), we determined concentrations of $489 \pm 107 \mathrm{nM}, 958 \pm 153 \mathrm{nM}$, and $859 \pm 81 \mathrm{nM}$. Such differences among biological replicates are not unusual when assaying c-di-AMP concentrations (Gundlach et al., 2015b). Importantly, our results demonstrate that M. pneumoniae does possess c-di-AMP, and imply the existence of enzymes that synthesize and degrade this second messenger.

To identify candidate genes involved in M. pneumoniae c-diAMP metabolism, we analyzed the genome for genes encoding proteins possessing a c-di-AMP synthesizing DAC domain as well as for proteins containing c-di-AMP degrading DHH-DHHA1 and $\mathrm{HD}$ domains. A single protein containing a DAC domain was found. The DAC domain of this protein, MPN244, is most similar to the domain of the B. subtilis sporulation-specific cyclase CdaS. However, the N-terminal domain of this protein is not similar to any domains found in other characterized diadenylate cyclases. According to the UniProt database, the amino acids 6

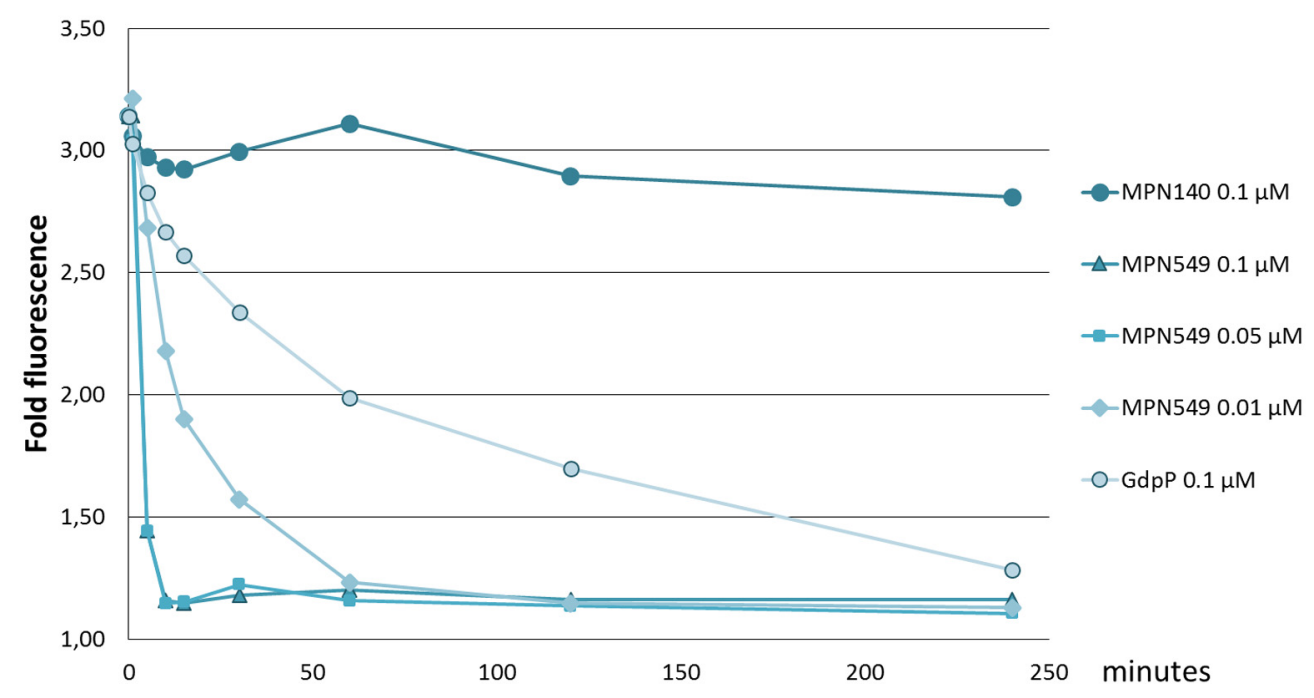

FIGURE 2 | Specific activity of phosphodiesterases toward c-di-AMP. Phosphodiesterases (MPN140, MPN549, GdpP: 100 nM, in addition 50 and 10 nM for MPN549) were added to $100 \mu \mathrm{M}$ c-di-AMP in $100 \mathrm{mM}$ Tris- $\mathrm{HCl}$ pH8.3, $10 \mathrm{mM} \mathrm{NaCl}, 0.1 \mathrm{mM} \mathrm{MnCl}$ at $37^{\circ} \mathrm{C}$. Reactions were stopped at 0, 5, 10, $15,30,60,120$, and 240 min. Upon addition of $\mathrm{KBr}$ and coralyne and excitation at $420 \mathrm{~nm}$, fluorescence at $475 \mathrm{~nm}$ was measured. The ratio of c-di-AMP-mediated emission vs. a standard without c-di-AMP is shown. Results from a typical experiment are shown. The replicates showed similar results. 
to 26 form a transmembrane helix. Thus, if MPN244 is endowed with diadenylate cyclase activity, this protein would represent a novel class of diadenylate cyclases. A comparison of the domain arrangement of the different classes of diadenylate cyclases is shown in Figure 1A.

An analysis of potential c-di-AMP degrading phosphodiesterases in $M$. pneumoniae failed to identify a protein containing a HD domain similar to that of the B. subtilis phosphodiesterase PgpH (Huynh et al., 2015). In contrast, the search for proteins containing a DHH-DHHA1 domain resulted in the identification of two proteins, MPN140 and MPN549. These proteins have been annotated as nano-RNase (NrnA) and probable single-stranded-DNA-specific exonuclease (RecJ) (Postic et al., 2012; Wodke et al., 2015), respectively. Two classes of c-di-AMP specific DHH-DHHAl phosphodiesterases have been identified: a class of large membrane-bound proteins, as exemplified by $B$. subtilis GdpP. This protein contains an N-terminal transmembrane domain, a PAS domain, and a degenerate GGDEF domain possibly involved in c-di-GMP sensing in addition to the DHH-DHHA1 domain. In contrast, the second class, represented by Pde2 of Streptococcus pneumoniae, consists only of the DHH-DHHA1 domain (Bai et al., 2013). Both potential phosphodiesterases of $M$. pneumoniae are similar to Pde2 rather than to GdpP (see Figure 1B for domain organization).

\section{CdaM (MPN244) Is a Diadenylate Cyclase}

In order to test whether MPN244 does indeed exhibit diadenylate cyclase activity, we took advantage of the inability of $E$. coli to produce c-di-AMP (Corrigan et al., 2011). The mpn244 gene was cloned into the expression vector $\mathrm{pET} 3 \mathrm{c}$, and the amounts of c-di-AMP produced in E. coli BL21(DE3) carrying the corresponding plasmid pGP2036, were compared to those of the same strain carrying the empty vector pET3c. As observed previously (Mehne et al., 2013), no c-di-AMP was present in the strain with the empty vector. In contrast, the expression of MPN244 resulted in the detection of $47.3 \pm 11.8 \mathrm{pmol}$ of $\mathrm{c}$-di-AMP/mg of protein. This result provides unequivocal evidence for the diadenylate cyclase activity of MPN244, and we

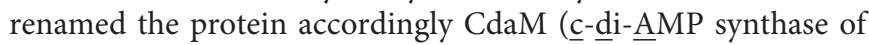
Mycoplasma), and the gene cdaM.

As c-di-AMP is essential in many Gram-positive bacteria, we wondered whether this is also the case in M. pneumoniae. To address this question, we attempted the isolation of a cdaM mutant from our transposon mutant library. This library contains 2,976 independent individual transposon mutants, and the probability to find mutants for any non-essential gene is as high as 99.999\% (Halbedel et al., 2006). In agreement with previous results (Lluch-Senar et al., 2015), no mutant could be isolated suggesting that the $c d a M$ gene and thus c-di-AMP is essential in M. pneumoniae.

\section{Phosphodiesterases Involved in the Degradation of c-di-AMP}

As mentioned above, two potential c-di-AMP degrading phosphodiesterases, MPN140 and MPN549, are encoded in the

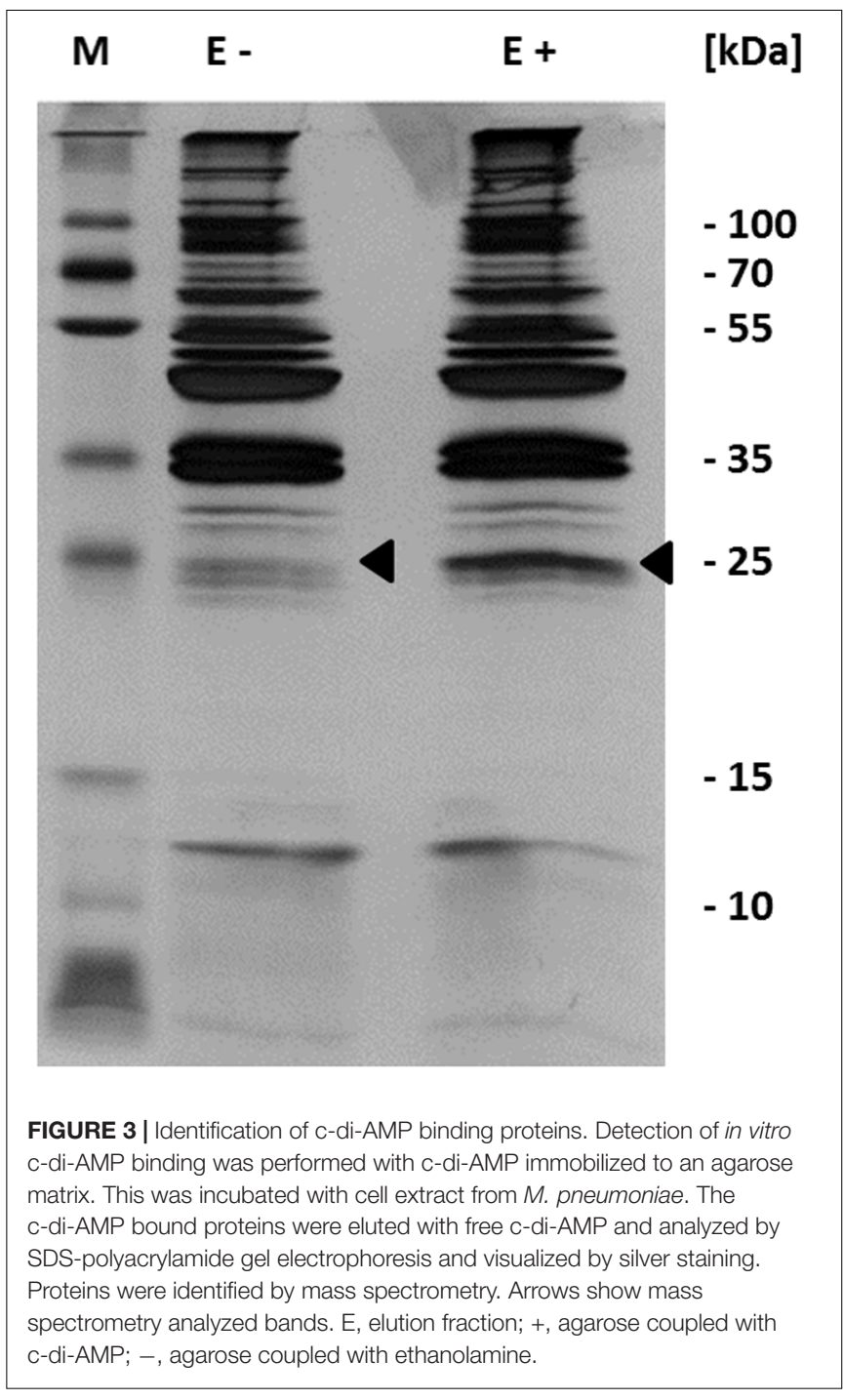

genome of $M$. pneumoniae. To assess their activity, we expressed and purified these enzymes carrying a C-terminal Strep-tag. Initially, we determined the phosphatase and phosphodiesterase activities of both enzymes. To determine phosphatase activities, we used $p$ NPP, a synthetic phosphatase substrate, and the M. pneumoniae protein phosphatase PrpC (Halbedel et al., 2006; Schmidl et al., 2010) as a positive control. While PrpC exhibited a strong phosphatase activity, only negligible activity was detected for MPN140 and MPN549 (data not shown). Phosphodiesterase activity was assayed against bis- $p N P P$ as the substrate, and the B. subtilis phosphodiesterase GdpP served as the control. As shown in Table 1, all three enzymes exhibited phosphodiesterase activity against this non-specific substrate. Thus, MPN140 and MPN549 are specifically active against phosphodiesters. In order to explore the activity of these enzymes against c-di-AMP, we determined c-di-AMP degradation using binding of c-di-AMP to the fluorescence dye coralyne as an assay. As shown in Figure 2, B. subtilis GdpP was capable of degrading the nucleotide. For MPN140, no activity against c-di-AMP was observed. In contrast, 


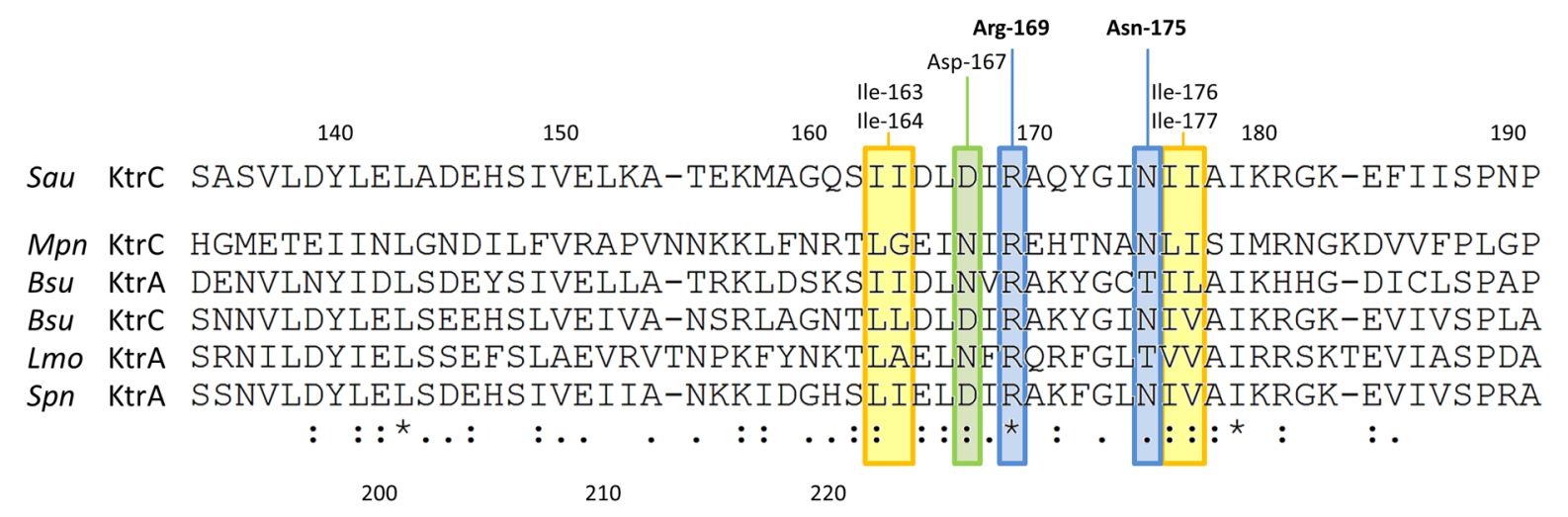

Sau KtrC NINLEIGDILIMIGHDNDLNRFEKNIATR---------

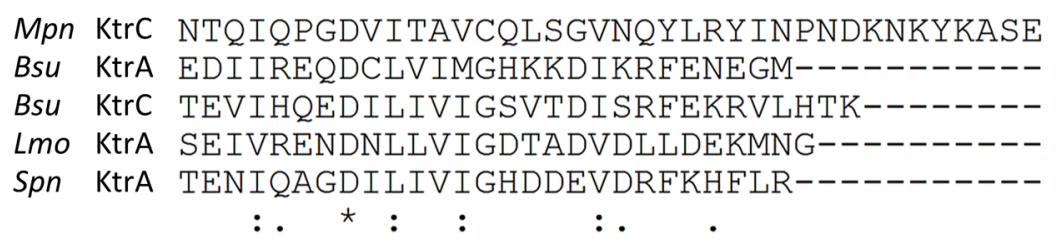

FIGURE 4 | Alignment of the c-di-AMP-binding regions in RCK_C domains of potassium uptake proteins. The C-terminal parts of different KtrC/ KtrA proteins were aligned to the experimentally identified c-di-AMP binding site of Staphylococcus aureus (Sau) KtrC (Kim et al., 2015). Residues responsible for c-di-AMP binding are highlighted: Arg-169 and Asn-175 (blue), which directly interact with c-di-AMP are conserved in M. pneumoniae KtrC; Asp-167 (green), which also forms a direct hydrogen bond with c-di-AMP is replaced by Asn which is also known to form hydrogen bonds, lle-163/lle-164 and lle-176/lle-177 (in yellow), which form the hydrophobic patch are well conserved in bacteria. Mpn Mycoplasma pneumoniae, Bsu Bacillus subtilis, Spn Streptococcus pneumoniae, Lmo Listeria monocytogenes. * identical amino acids, : similar amino acids.

MPN549 exhibited high activity against c-di-AMP. As little as $10 \%$ of the enzyme amount used in the assay with GdpP degraded the nucleotide more rapidly than GdpP. To conclude, our results clearly demonstrate that MPN140 is a phosphodiesterase, but with a substrate distinct from c-di-AMP whereas MPN549 is the c-di-AMP-degrading phosphodiesterase in M. pneumoniae. Thus, MPN549 was renamed PdeM (phosphodiesterase of $\underline{M}$. pneumoniae), and the gene accordingly $p d e M$.

In B. subtilis, c-di-AMP is not only essential but also toxic in the absence of the phosphodiesterases that degrade the nucleotide (Gundlach et al., 2015b). Thus, we tested whether a pdeM mutant could be isolated from our transposon mutant collection. As observed in a global transposon mutant study (Lluch-Senar et al., 2015), no mutant could be isolated indicating that the pdeM gene is essential in M. pneumoniae.

\section{Identification of the c-di-AMP Target Protein KtrC (MPN461)}

Our results indicate that c-di-AMP is synthesized in M. pneumoniae, and that this second messenger is essential for the growth of the bacteria. This suggests an important physiological function of c-di-AMP. To identify this function, we decided to isolate c-di-AMP-binding proteins, which are excellent candidates for c-di-AMP-mediated regulation. For this purpose, we used c-di-AMP immobilized via a linker to agarose and incubated it with a protein extract of $M$. pneumoniae. As a control, we used agarose containing ethanolamine immobilized via the same linker. After extensive washing (see Materials and Methods), c-di-AMP-binding proteins were eluted with free c-diAMP. The proteins were analyzed by SDS-PAGE (see Figure 3), and a band exhibiting increased intensity in the binding assay identified by mass spectrometry as MPN461. This protein is similar to the $B$. subtilis low affinity potassium transporter regulatory subunit KtrC (Holtmann et al., 2003). The mpn461 gene forms a gene cluster with the upstream gene $m p n 460$, which encodes a protein most similar to the integral membrane subunit $\mathrm{KtrD}$ of the KtrC-KtrD potassium channel. An alignment of the c-di-AMP-binding region of S. aureus (Kim et al., 2015) with the relevant residues of $M$. pneumoniae and related proteins from other bacteria indicates that the amino acids interacting with c-di-AMP are conserved in the M. pneumoniae protein (see Figure 4). Taken together, these results suggest that c-di-AMP controls potassium transport in M. pneumoniae.

\section{DISCUSSION}

In this work, we provide the first analysis of c-di-AMP signaling in the genome-reduced bacterium $M$. pneumoniae. We have identified the diadenylate cyclase CdaM as well as the c-diAMP degrading phosphodiesterase PdeM. Moreover, we have demonstrated binding of $\mathrm{c}$-di-AMP to $\mathrm{KtrC}$, the regulatory subunit of a potassium transporter.

So far, three distinct classes of diadenylate cyclases have been characterized. The M. pneumoniae CdaM protein is the prototype 
of a fourth class that is specific to the genome-reduced members of the genus Mycoplasma. The domains present in the diadenylate cyclases in addition to the enzymatically active DAC domain are thought to be required for signal recognition by the cyclases and thereby to control the activity of the enzymes (Mehne et al., 2014; Commichau et al., 2015). Thus, it is tempting to speculate that the single transmembrane domain associated with CdaM reflects the specific lifestyle of the highly host-dependent and host-adapted Mycoplasma cells. Taking into account the highly peculiar physiology and cell biology of the Mycoplasmas, it seems likely that the organization of CdaM is an adaptation to this particular biology.

The phosphodiesterase PdeM consists of a single DHH-DHHA1 domain. This type of c-di-AMP-specific phosphodiesterases has been found in other pathogenic bacteria, including S. pneumoniae, Borrelia burgdorferi, and Mycobacterium tuberculosis. While S. pneumoniae encodes both a classical phosphodiesterase (similar to GdpP, see Figure 1), the latter bacteria possess only the one-domain proteins, as observed in M. pneumoniae (Bai et al., 2013; Manikandan et al., 2014; Ye et al., 2014). In addition to PdeM, M. pneumoniae possesses a very similar protein, NrnA (MPN140). The two proteins share $29 \%$ identity, which is indicative of functional specialization. Indeed, while both proteins exhibit phosphodiesterase activity toward a generic substrate, only PdeM is able to degrade c-diAMP. This is in good agreement with the reported activity of NrnA toward short oligoribonucleotides (Postic et al., 2012). Based on the results obtained with other cytoplasmic c-di-AMP specific phosphodiesterases (Bai et al., 2013; Manikandan et al., 2014; Ye et al., 2014), it is tempting to speculate that PdeM may degrade c-di-AMP to pApA, which is in turn degraded to AMP by the activity of NrnA. It is interesting to note that the presence of closely related proteins of which only one has the suspected biochemical activity is not unprecedented in M. pneumoniae: of the two potential glycerophosphate phosphodiesterases, only one protein (GlpQ) has the corresponding enzymatic activity, whereas no function has so far been identified for the second protein (MPN566) (Schmidl et al., 2011).

While the reason for c-di-AMP essentiality has long been enigmatic, recent research has suggested that the control of potassium homeostasis is the essential function (Gundlach et al., 2017). Three lines of evidence support this idea: (i) c-di-AMP controls potassium uptake in B. subtilis, L. monocytogenes, and $S$. aureus both at the level of transporter activity and transporter expression, and it does so by binding very different classes of proteins and even RNA. (ii) In B. subtilis, c-di-AMP becomes non-essential if the bacteria are cultivated at low potassium concentrations. (iii) The control of potassium uptake is the common denominator of c-di-AMP signaling in all organisms that have been studied in this respect. The demonstration that even in the genome-reduced bacterium M. pneumoniae c-di$\mathrm{AMP}$ binds to the regulatory subunit of the KtrCD potassium transporter is in strong support for a general involvement of c-di-AMP in the control of potassium homeostasis. Moreover, the essentiality of both the diadenylate cyclase CdaM and the phosphodiesterase PdeM indicates that essentiality is not derived from the control of a single protein, but that it is rather related to a homeostatic process that is essential for the cell but that needs to be limited. This reflects the nature of potassium which is essential for any living cell but which becomes toxic if it accumulates in the cell to non-physiological concentrations (Nissen et al., 2000; Gundlach et al., 2017).

The essentiality of c-di-AMP synthesizing and degrading enzymes and the absence of c-di-AMP and c-di-AMP metabolizing enzymes from human cells makes these proteins excellent candidates for the development of novel antimicrobial drugs. Our work clearly suggests an important function for cyclic di-AMP in M. pneumoniae, but further work will be required to understand the molecular mechanisms by which the activity of $\mathrm{CdaM}$ is controlled as well as the regulation of KtrC activity by c-di-AMP.

\section{AUTHOR CONTRIBUTIONS}

$\mathrm{CB}, \mathrm{KT}$, and JS designed the study. $\mathrm{CB}$ and $\mathrm{KT}$ performed the experiments. VK quantified the nucleotides. FS prepared the c-diAMP-coupled agarose. EH identified the proteins. $\mathrm{CB}$ and JS wrote the paper.

\section{FUNDING}

This research received funding from the Federal Ministry of Education and Research MiniCell network (Grant 031L0012). Katrin Treffon was supported by a Dorothea-Schlözer stipend from the Georg-August-University of Göttingen.

\section{ACKNOWLEDGMENTS}

We are grateful to Julia Busse and Annette Garbe for excellent technical assistance and to Tobias Krammer for the help with the characterization of phosphodiesterases. We wish to thank Hinnerk Eilers and Arne Schmeisky for having initiated this project. Achim Dickmanns and Katrin Gunka are acknowledged for helpful discussions.

\section{SUPPLEMENTARY MATERIAL}

The Supplementary Material for this article can be found online at: http://journal.frontiersin.org/article/10.3389/fmicb. 2017.01328/full\#supplementary-material 


\section{REFERENCES}

Bai, Y., Yang, J., Eisele, L. E., Underwood, A. J., Koestler, B. J., Waters, C. M., et al. (2013). Two DHH subfamily 1 proteins in Streptococcus pneumoniae possess cyclic di-AMP phosphodiesterase activity and affect bacterial growth and virulence. J. Bacteriol. 195, 5123-5132. doi: 10.1128/JB.00769-13

Campos, S. S., Ibarra-Rodriguez, J. R., Barajas-Ornelas, R. C., Ramírez-Guadiana, F. H., Obregón-Herrera, A., Setlow, P., et al. (2014). Interaction of apurinic/ apyrimidinic endonucleases Nfo and ExoA with the DNA integrity scanning protein DisA in the processing of oxidative DNA damage during Bacillus subtilis spore outgrowth. J. Bacteriol. 196, 568-578. doi: 10.1128/JB.01259-13

Commichau, F. M., Dickmanns, A., Gundlach, J., Ficner, R., and Stülke, J. (2015). A jack of all trades: the multiple roles of the unique essential second messenger cyclic di-AMP. Mol. Microbiol. 97, 189-204. doi: 10.1111/mmi.13026

Corrigan, R. M., Abbott, J. C., Burhenne, H., Kaever, V., and Gründling, A. (2011). c-di-AMP is a new second messenger in Staphylococcus aureus with a role in controlling cell size and envelope stress. PLoS Pathog. 7:e1002217. doi: 10.1371/ journal.ppat.1002217

Corrigan, R. M., Campeotto, I., Jeganathan, T., Roelofs, K. G., Lee, V. T., and Gründling, A. (2013). Systematic identification of conserved bacterial c-di-AMP receptor proteins. Proc. Natl. Acad. Sci. U.S.A. 110, 9084-9089. doi: 10.1073/pnas.1300595110

Corrigan, R. M., and Gründling, A. (2013). Cyclic di-AMP: another second messenger enters the fray. Nat. Rev. Microbiol. 11, 513-524. doi: 10.1038/ nrmicro3069

Diethmaier, C., Newman, J. A., Kovács, ÁT., Kaever, V., Herzberg, C., Rodrigues, C., et al. (2014). The YmdB phosphodiesterase is a global regulator of late adaptive responses in Bacillus subtilis. J. Bacteriol 196, 265-275. doi: 10.1128/JB.00826-13

Eilers, H. (2010). Transcription in Mycoplasma pneumoniae. Ph.D. thesis, GeorgAugust-University of Göttingen, Göttingen.

Gomelsky, M. (2011). cAMP, c-di-GMP, c-di-AMP and now cGMP: bacteria use them all! Mol. Microbiol. 79, 562-565. doi: 10.1111/j.1365-2958.2010.07514.x

Görke, B., and Stülke, J. (2008). Carbon catabolite repression in bacteria: many ways to make most out of nutrients. Nat. Rev. Microbiol. 6, 613-624. doi: 10.1038/nrmicro1932

Güell, M., van Noort, V., Yus, E., Chen, W. H., Leigh-Bell, J., Michalodimitrakis, K., et al. (2009). Transcriptome complexity in a genome-reduced bacterium. Science 326, 1268-1271. doi: 10.1126/science.1176951

Gundlach, J., Dickmanns, A., Schröder-Tittmann, K., Neumann, P., Kaesler, J., Kampf, J., et al. (2015a). Identification, characterization, and structure analysis of the cyclic di-AMP-binding PII-like signal transduction protein DarA. J. Biol. Chem. 290, 3069-3080. doi: 10.1074/jbc.M114.619619

Gundlach, J., Herzberg, C., Kaever, V., Gunka, K., Hoffmann, T., Weiß, M., et al. (2017). Control of potassium homeostasis is an essential function of the second messenger cyclic di-AMP in Bacillus subtilis. Sci. Signal. 10:eaal3011. doi: 10.1126/scisignal.aal3011

Gundlach, J., Mehne, F. M., Herzberg, C., Kampf, J., Valerius, O., Kaever, V., et al. (2015b). An essential poison: synthesis and degradation of cyclic di-AMP in Bacillus subtilis. J. Bacteriol. 197, 3265-3274. doi: 10.1128/JB.00564-15

Halbedel, S., Busse, J., Schmidl, S., and Stülke, J. (2006). Regulatory protein phosphorylation in Mycoplasma pneumoniae: a PP2C-type phosphatase serves to dephosphorylate $\mathrm{HPr}(\mathrm{Ser}-\mathrm{P})$. J. Biol. Chem. 281, 26253-26259. doi: 10.1074/ jbc.M605010200

Halbedel, S., Hames, C., and Stülke, J. (2004). In vivo activity of enzymatic and regulatory components of the phosphoenolpyruvate:sugar phosphotransferase system in Mycoplasma pneumoniae. J. Bacteriol. 186, 7936-7943. doi: 10.1128/ JB.186.23.7936-7943.2004

Hames, C., Halbedel, S., Schilling, O., and Stülke, J. (2005). MMR: a method for the simultaneous introduction of multiple mutations into the glpK gene of Mycoplasma pneumoniae. Appl. Environ. Microbiol. 71, 4097-4100. doi: 10. 1128/AEM.71.7.4097-4100.2005

Hengge, R. (2009). Principles of c-di-GMP signaling in bacteria. Nat. Rev. Microbiol. 7, 263-273. doi: 10.1038/nrmicro2109

Holtmann, G., Bakker, E. P., Uozumi, N., and Bremer, E. (2003). KtrAB and KtrCD: two $\mathrm{K}+$ uptake systems in Bacillus subtilis and their role in adaptation to hypertonicity. J. Bacteriol. 185, 1289-1298. doi: 10.1128/JB.185.4.1289-1298. 2003
Huynh, T. N., Choi, P. H., Sureka, K., Ledvina, H. E., Campillo, J., Tong, L., et al. (2016). Cyclic di-AMP targets the cystathionine beta-synthase domain of the osmolyte transporter OpuC. Mol. Microbiol. 102, 233-243. doi: 10.1111/mmi. 13456

Huynh, T. N., Luo, S., Pensinger, D., Sauer, J. D., Tong, L., and Woodward, J. J. (2015). An HD-domain phosphodiesterase mediates cooperative hydrolysis of c-di-AMP to affect bacterial growth and virulence. Proc. Natl. Acad. Sci. U.S.A. 112, E747-E756. doi: 10.1073/pnas.1416485112

Huynh, T. N., and Woodward, J. J. (2016). Too much of a good thing: regulated depletion of c-di-AMP in the bacterial cytoplasm. Curr. Opin. Microbiol. 30, 22-29. doi: 10.1016/j.mib.2015.12.007

Kim, H., Youn, S. J., Kim, S. O., Ko, J., Lee, J. O., and Choi, B. S. (2015). Structural studies of potassium transport protein KtrA regulator of conductance of $\mathrm{K}+$ (RCK) C domain in complex with cyclic diadenosine monophosphate (c-di-AMP). J. Biol. Chem. 290, 16393-16402. doi: 10.1074/jbc.M115. 641340

Lluch-Senar, M., Delgado, J., Chen, W. H., Lloréns-Rico, V., O’Reilly, F. J., Wodke, J. A., et al. (2015). Defining a minimal cell: essentiality of small ORFs and ncRNAs in a genome-reduced bacterium. Mol. Syst. Biol. 11:780. doi: 10.15252/ msb. 20145558

Luo, Y., and Helmann, J. D. (2012). Analysis of the role of Bacillus subtilis $\sigma \mathrm{M}$ in $\beta$-lactam resistance reveals an essential role for c-di-AMP in peptidoglycan homeostasis. Mol. Microbiol. 83, 623-639. doi: 10.1111/j.1365-2958.2011. 07953.x

Manikandan, K., Sabareesh, V., Singh, N., Saigal, K., Mechold, U., and Sinha, K. M. (2014). Two-step synthesis and hydrolysis of cyclic di-AMP in Mycobacterium tuberculosis. PLoS ONE 9:e86096. doi: 10.1371/journal.pone.0086096

Mehne, F. M., Schröder-Tittmann, K., Eijlander, R. T., Herzberg, C., Hewitt, L., Kaever, V., et al. (2014). Control of the diadenylate cyclase CdaS in Bacillus subtilis: an autoinhibitory domain limits c-di-AMP production. J. Biol. Chem. 289, 21098-21107. doi: 10.1074/jbc.M114.562066

Mehne, F. M. P., Gunka, K., Eilers, H., Herzberg, C., Kaever, V., and Stülke, J. (2013). Cyclic-di-AMP homeostasis in Bacillus subtilis: both lack and high-level accumulation of the nucleotide are detrimental for cell growth. J. Biol. Chem. 288, 2004-2017. doi: 10.1074/jbc.M112.395491

Meyer, F. M., Gerwig, J., Hammer, E., Herzberg, C., Commichau, F. M., Völker, U., et al. (2011). Physical interactions between tricarboxylic acid cycle enzymes in Bacillus subtilis: evidence for a metabolon. Metab. Eng. 13, 18-27. doi: 10.1016/ j.ymben.2010.10.001

Moscoso, J. A., Schramke, H., Zhang, Y., Tosi, T., Dehbi, A., Jung, K., et al. (2016). Binding of cyclic di-AMP tot he Staphylococcus aureus sensor kinase $\mathrm{KdpD}$ occurs via the universal stress protein domain and downregulates the expression of the Kdp potassium transporter. J. Bacteriol. 198, 98-110. doi: 10.1128/JB.00480-15

Nelson, J. W., Sudarsan, N., Furukawa, K., Weingerg, Z., Wang, J. X., and Breaker, R. R. (2013). Riboswitches in eubacteria sense the second messenger cyclic di-AMP. Nat. Chem. Biol. 9, 834-839. doi: 10.1038/nchembio. 1363

Nissen, P., Hansen, J., Ban, N., Moore, P. B., and Steitz, T. A. (2000). The structural basis of ribosome activity in peptide bond synthesis. Science 289, 920-930. doi: $10.1126 /$ science.289.5481.920

Oppenheimer-Shaanan, Y., Wexselblatt, E., Katzhaendler, J., Yavin, E., and BenYehuda, S. (2011). c-di-AMP reports DNA integrity during sporulation in Bacillus subtilis. EMBO Rep. 12, 594-601. doi: 10.1038/embor.2011.77

Postic, G., Danchin, A., and Mechold, U. (2012). Characterization of NrnA homologs from Mycobacterium tuberculosis and Mycoplasma pneumoniae. RNA 18, 155-165. doi: 10.1261/rna.029132.111

Rao, F., See, R. Y., Zhang, D., Toh, D. C., Ji, Q., and Liang, Z. X. (2010). YybT is a signaling protein that contains a cyclic dinucleotide phosphodiesterase domain and a GGDEF domain with ATPase activity. J. Biol. Chem. 285, 473-482. doi: $10.1074 /$ jbc.M109.040238

Römling, U. (2008). Great times for small molecules: c-di-AMP, a second messenger candidate in bacteria and archaea. Sci. Signal. 1:e39. doi: 10.1126/ scisignal.133pe39

Rosenberg, J., Dickmanns, A., Neumann, P., Gunka, K., Arens, J., Kaever, V., et al. (2015). Biochemical and structural analysis of the essential diadenylate cyclase CdaA from Listeria monocytogenes. J. Biol. Chem. 290, 6596-6606. doi: $10.1074 /$ jbc.M114.630418 
Sambrook, J., Fritsch, E. F., and Maniatis, T. (1989). Molecular Cloning: A Laboratory Manual, 2nd Edn. Cold Spring Harbor, NY: Cold Spring Harbor Laboratory.

Schilling, O., Herzberg, C., Hertrich, T., Vörsmann, H., Jessen, D., Hübner, S., et al. (2006). Keeping signals straight in transcription regulation: specificity determinants for the interaction of a family of conserved bacterial RNA-protein couples. Nucleic Acids Res. 34, 6102-6115. doi: 10.1093/nar/gkl733

Schmidl, S. R., Gronau, K., Hames, C., Busse, J., Becher, D., Hecker, M., et al. (2010). The stability of cytadherence proteins in Mycoplasma pneumoniae requires activity of the protein kinase PrkC. Infect. Immun. 78, 184-192. doi: 10.1128/ IAI.00958-09

Schmidl, S. R., Otto, A., Lluch-Senar, M., Piñol, J., Busse, J., Becher, D., et al. (2011). A trigger enzyme in Mycoplasma pneumoniae: impact of the glycerophosphodiesterase GlpQ on virulence and gene expression. PLOS Pathogens 7:e1002263. doi: 10.1371/journal.ppat.1002263

Schuster, C. F., Bellows, L. E., Tosi, T., Campeotto, I., Corrigan, R. M., Freemont, P., et al. (2016). The second messenger c-di-AMP inhibits the osmolyte uptake system OpuC in Staphylococcus aureus. Sci. Signal. 9:ra81. doi: 10.1126/ scisignal.aaf7279

Steinchen, W., and Bange, G. (2016). The magic dance of the alarmones (p)ppGpp. Mol. Microbiol. 101, 531-544. doi: 10.1111/mmi.13412

Sureka, K., Choi, P. H., Precit, M., Delince, M., Pensinger, D. A., Huynh, T. N., et al. (2014). The cyclic dinucleotide c-di-AMP is an allosteric regulator of metabolic enzyme function. Cell 158, 1389-1401. doi: 10.1016/j.cell.2014.07.046

Witte, G., Hartung, S., Büttner, K., and Hopfner, K. P. (2008). Structural biochemistry of a bacterial checkpoint protein reveals diadenylate cyclase activity regulated by DNA recombination intermediates. Mol. Cell 30, 167-178. doi: 10.1016/j.molcel.2008.02.020
Wodke, J. A., Alibés, A., Cozzuto, L., Hermoso, A., Yus, E., Lluch-Senar, M., et al. (2015). MyMpn: a database for the systems biology model organism Mycoplasma pneumoniae. Nucleic Acids Res. 43, D618-D623. doi: 10.1093/nar/ gku1105

Ye, M., Zhang, J. J., Fang, X., Lawlis, G. B., Troxell, B., Zhou, Y., et al. (2014). DhhP, a cyclic di-AMP phosphodiesterase of Borrelia burgdorferi, is essential for cell growth and virulence. Infect. Immun. 82, 1840-1849. doi: 10.1128/IAI. 00030-14

You, C., Okano, H., Hui, S., Zhang, Z., Kim, M., Gunderson, C. W., et al. (2013). Coordination of bacterial proteome with metabolism by cyclic AMP signaling. Nature 500, 301-306. doi: 10.1038/nature12446

Zhou, J., Sayre, D. A., Zheng, Y., Szmacinski, H., and Sintim, H. O. (2014). Unexpected complex formation between coralyne and c-diAMP provides a simple fluorescent turn-on assay to detect this bacterial second messenger. Anal. Chem. 86, 2412-2420. doi: 10.1021/ac403 $203 x$

Conflict of Interest Statement: The authors declare that the research was conducted in the absence of any commercial or financial relationships that could be construed as a potential conflict of interest.

Copyright () 2017 Blötz, Treffon, Kaever, Schwede, Hammer and Stülke. This is an open-access article distributed under the terms of the Creative Commons Attribution License (CC BY). The use, distribution or reproduction in other forums is permitted, provided the original author(s) or licensor are credited and that the original publication in this journal is cited, in accordance with accepted academic practice. No use, distribution or reproduction is permitted which does not comply with these terms. 